\title{
Condições de vida e saúde de idosos com transtornos mentais de acordo com o sexo*
}

\author{
Maria Cássia Corrêa Mazzi Freire ${ }^{1}$ \\ (D) https://orcid.org/0000-0002-3264-3153 \\ Maria José Sanches Marin 1 \\ (D) https://orcid.org/0000-0001-6210-6941 \\ Carlos Alberto Lazarini ${ }^{1}$ \\ (D) https://orcid.org/0000-0003-3010-4436 \\ Daniela Garcia Damaceno² \\ (D) http://orcid.org/0000-0001-8656-009x
}

\begin{abstract}
Objetivo: analisar as condições de vida e saúde de idosos usuários de ambulatório de saúde mental de acordo com o sexo. Método: estudo transversal realizado em Ambulatório de Saúde Mental do interior paulista, Brasil, com 138 idosos, por meio de entrevistas. As análises inferenciais foram realizadas por meio do teste de qui-quadrado de Pearson e da extensão do teste exato de Fisher. Resultados: as mulheres realizavam mais frequentemente prevenção de câncer, vacinação da gripe, uso de aparelhos auxiliares, quando necessários; apresentaram diagnósticos em saúde mental realizados na vida adulta, estado conjugal "vive só", independência nas atividades de vida diária e transtornos de humor e de personalidade. Os homens tiveram diagnósticos em saúde mental mais tardios, uso pregresso de álcool e transtornos mentais e de comportamento decorrentes do uso de substâncias psicoativas. Conclusão: as condições de vida e saúde dos idosos com transtorno mental apresentaram características diferentes quando se comparou o sexo masculino com o feminino. Isto evidencia a necessidade da busca pelo conhecimento das diferenças, para quem os atende profissionalmente, considerando os contextos que acarretaram o adoecimento e as condições atuais de saúde.
\end{abstract}

Descritores: Condições Sociais; Saúde Mental; Idoso;

Nível de Saúde.

\section{Como citar este artigo}

Freire MCCM, Marin MJS, Lazarini CA, Damaceno DG. Life and health conditions of the elderly with mental disorders according to sex. SMAD, Rev Eletrônica Saúde Mental Álcool Drog. 2020;16(1):1-11. doi: https://dx.doi.org/10.11606/issn.1806-6976.smad.2020.153846 


\section{Life and health conditions of the elderly with mental disorders according to sex}

Objective: to analyze the conditions of life and health of elderly users of an outpatient mental health facility in accordance with the gender. Method: a cross-sectional study conducted in an outpatient mental health facilities in the countryside of São Paulo, with 138 elderly, through interviews. The inferential analyzes were performed by using the Pearson Chi-square test and the extension of the Fishers exact test. Results: women are more often the prevention of cancer, flu vaccination, use of auxiliary aids when necessary; present diagnoses in mental health carried out in adult life, marital status "lives", independence in activities of daily living and mood disorders and personality. The men had their diagnoses in mental health later, use habit of alcohol and mental and behavioral disorders due to use of psychoactive substance. Conclusion: the living conditions and health of elderly people with mental disorder have different characteristics when comparing males with females. This evidences the need for the search for knowledge of the differences, for those who attend them professionally, considering the contexts that led to illness and current health conditions.

Descriptors: Social Conditions; Mental Health; Elderly; Health Status.

\section{Condiciones de vida y salud de los ancianos con trastornos mentales, según el sexo}

Objetivo: analizar las condiciones de vida y salud de ancianos usuarios de ambulatorio de salud mental de acuerdo con el sexo. Método: estudio transversal, llevado a cabo en Ambulatorio de Salud Mental del interior paulista, Brasil, con 138 ancianos, por medio de entrevistas. Los análisis inferenciales se realizaron por medio de la Prueba de Qui-cuadrado de Pearson y la extensión de la prueba Exacto de Fisher. Resultados: las mujeres realizaban más frecuentemente la prevención de cáncer, vacunación de la gripe, uso de aparatos auxiliares, cuando sea necesario; que presentan diagnósticos en salud mental realizados en la vida adulta, estado conyugal "vive solo", independencia en las actividades de vida diaria y trastornos de humor y de personalidad. Los hombres tuvieron diagnósticos en salud mental más tardíos, uso progresó de alcohol y trastornos mentales y de comportamiento derivados del uso de sustancia psicoactiva. Conclusión: las condiciones de vida y salud de los ancianos con trastorno mental presentaran características diferentes, cuando se comparó el sexo masculino con el femenino. Esto evidencia la necesidad de la búsqueda por el conocimiento de las diferencias, para quien los atiende profesionalmente, considerando los contextos que llevaron al enfermo y a las condiciones actuales de salud.

Descriptores: Condiciones Sociales; Salud Mental; Anciano; Estado de Salud. 


\section{Introdução}

No Brasil, há décadas, o processo de envelhecimento vem sendo colocado em pauta por estudiosos do assunto, que apontam para consequências do aumento do número de idosos, sem acréscimo na qualidade de vida. Essa população apresenta inúmeros problemas de saúde, sendo necessário maior cuidado, especialmente no caso de idosos portadores de transtornos mentais.

Estudo europeu estimou que um em cada dois idosos haviam experimentado um transtorno mental durante a vida, sendo um em cada três, no ano que antecedeu à pesquisa, e um em cada quatro no momento da entrevista. Os distúrbios mais comuns foram transtornos da ansiedade seguidos de perturbações afetivas e relacionadas ao uso de substâncias psicoativas. Logo, é preciso refinamento no diagnóstico de transtorno mental entre idosos e fornecimento de serviços de saúde mental de qualidade a estes ${ }^{(1)}$.

Estudo nacional, realizado no interior do Estado de São Paulo, Brasil, com 219 idosos atendidos pela Estratégia Saúde da Família, identificou que 19,6\% apresentaram escores acima de sete, ou seja, com indicativo da presença de transtorno mental(2).

$\mathrm{Na}$ atualidade, a assistência à pessoa com transtorno mental é realizada predominantemente em base comunitária. As Políticas de Atenção à Saúde Mental que, a partir de 1978, passaram pelo movimento da Reforma Psiquiátrica, orientam, entre outras ações, a desospitalização, resultando na criação de cenários, como os Centros de Atenção Psicossocial (CAPS), o Programa de Volta para Casa e o apoio matricial, com especialistas da saúde mental às equipes de atenção básica. Além desses, nos anos de 1980, foram criados os ambulatórios especializados em saúde mental, como dispositivos da reforma, serviços complementares que atuam na continuidade do tratamento(3).

Com as mudanças das práticas de intervenção psiquiátrica, iniciadas com a reorganização do modelo de assistência aos doentes mentais nos anos de 1980, houve redução de $44,8 \%$ no número de leitos em hospitais psiquiátricos. As internações psiquiátricas diminuíram em cerca de 35\%, no período entre 1996 e 2005, porém, nesse período, houve aumento de $89,3 \%$ da mortalidade de pacientes com diagnósticos de transtornos mentais ${ }^{(4)}$.

Sobre a qualidade dos serviços ambulatoriais produzidos após o movimento da Reforma Psiquiátrica, estudo do Conselho Regional de Medicina do Estado de São Paulo apontou falhas no funcionamento dos CAPS, como a escassez de retaguarda para internações psiquiátricas e emergências, especialmente carência de profissionais médicos, entre outras, o que prejudica o atendimento em saúde mental ${ }^{(4)}$. Inexistem, também, estudos mais esclarecedores sobre ambulatórios especializados em Saúde Mental das instituições de ensino.
Sabe-se que $40 \%$ dos países membros das Nações Unidas não possuem política de saúde mental. Além disso, o cuidado com as pessoas idosas não é considerado prioridade em grande parte dos países do mundo(5). Idosos com transtornos mentais, adquiridos na idade adulta ou velhice, apresentam condições de vida e saúde com grande complexidade, o que exige adequado reconhecimento para que ações possam ser direcionadas a essa parcela da população.

Além disso, é preciso considerar que, entre os idosos com transtornos mentais, existem questões relacionadas ao sexo, uma vez que homens e mulheres são obrigados a assumir posição social e historicamente construída, que constitui fator determinante das condições de vida e saúde(6).

As condições de vida e saúde, portanto, remetem ao conceito ampliado de saúde proposto pela Constituição Federal de 1988. Envolve os determinantes sociais que se traduzem em condições mais gerais, como as socioeconômicas, culturais e ambientais, incluindo condições de trabalho, habitação, saneamento, serviços de saúde, educação, redes sociais e comunitárias, as quais determinam o modo de vida das pessoas e se configuram como produtoras da saúde e das formas de adoecer e morrer( ${ }^{(7)}$.

Ao considerar que a equidade em saúde, enquanto um princípio garantido na Constituição, é reconhecida a partir da delimitação das necessidades de grupos específicos, este estudo teve como questão norteadora: "Quais as condições de vida e saúde de idosos portadores de transtornos mentais, de acordo com o sexo?".

Frente a esse contexto, objetivou-se analisar as condições de vida e saúde de idosos usuários de ambulatório de saúde mental de acordo com o sexo.

\section{Método}

Estudo transversal, descritivo e analítico, realizado em Ambulatório de Saúde Mental de Instituição de Ensino Superior, do centro-oeste do Estado de São Paulo, Brasil, com idosos portadores de transtorno mental.

O tamanho de amostra foi calculado utilizando-se dos seguintes parâmetros estatísticos: população idosa que frequenta o ambulatório de saúde mental, mensalmente, em média $=208$ (dado fornecido pelo Núcleo de Tecnologia e Informação da instituição); erro amostral de $5 \%$; nível de confiança de $95 \%$ e percentual máximo de 50\%, necessitando-se de 138 indivíduos. Foram incluídos na amostra os idosos que apresentaram capacidade cognitiva, visual e auditiva para responder ao questionário, que possuíam, pelo menos, um diagnóstico confirmado de transtorno mental, excluindo-se os dependentes de cuidadores.

Para a coleta de dados, o questionário utilizado pautou-se no projeto Saúde, Bem-estar e Envelhecimento $(\mathrm{SABE})^{(8)}$, que conta com dados sociodemográficos, 
incluindo sexo, idade, escolaridade, estado conjugal, religião; o Critério de Classificação Econômica Brasil, idade da aposentadoria, participação econômica dentro da renda familiar, origem da renda, dados de características da residência e do saneamento básico. Sobre as condições de saúde, foram coletados dados referentes às condições de comunicação, autopercepção da saúde, uso de álcool, vacina da gripe no último ano, prevenção de câncer de mama/colo/próstata, condições de audição, visão, mastigação, uso de aparelhos auxiliares e quedas.

Além disso, os idosos foram interrogados quanto à presença dos principais sinais e sintomas e doenças presentes no envelhecimento; diagnóstico médico do transtorno mental (obtido a partir de consulta ao prontuário médico), idade em que o diagnóstico foi realizado, número de internações no último ano e acesso ao tratamento em saúde mental. Para avaliar o grau de dependência, foi utilizada a Escala de Lawton \& Brody ${ }^{(9)}$. Definiu-se o sexo como variável independente. As demais foram tratadas como variáveis dependentes. Os dados foram coletados pela pesquisadora principal, de janeiro a março de 2016.

As análises estatísticas foram realizadas com o software SPSS, versão 17.0. As análises inferenciais foram realizadas por meio do teste de qui-quadrado de Pearson e da extensão do teste exato de Fisher. Em todas as conclusões obtidas pelas análises inferenciais, utilizou-se do nível de significância a igual a 5\% ( $p \leq 0,05)$.

O estudo foi aprovado pelo Comitê de Ética em Pesquisa envolvendo Seres Humanos da Faculdade de Medicina de Marília, seguindo a Resolução 466 de 12/12/2012 do Conselho Nacional de Saúde, conforme o número 1.050.605. Os participantes foram informados pelo entrevistador sobre a natureza do estudo, objetivos, métodos e benefícios previstos, potenciais riscos e possíveis incômodos pelo entrevistador e, para participar, assinaram o Termo de Consentimento Livre e Esclarecido (TCLE).

\section{Resultados}

Dos 138 entrevistados, 47 eram homens e 91, mulheres. Ambos os sexos apresentaram predomínio de idade entre 60 e 69 anos e séries iniciais incompletas ( $1^{a}$ a $4^{a}$ séries) [a Legislação não utIliza fundamental I e II, somente fundamental, sugiro colocar séries iniciais] enquanto escolaridade. Para o estado conjugal, houve tendência para a diferença estatisticamente significativa entre os sexos $(p=0,06)$, sendo que as mulheres apontaram a opção "vive sem companheiro" em maior proporção do que os homens. A religião católica foi a mais presente entre os entrevistados. Os homens declararam-se católicos em maior proporção do que as mulheres $(p=0,02)$ (Tabela 1$)$.

Tabela 1 - Distribuição das principais características sociodemográficas dos idosos atendidos no Ambulatório de Saúde Mental segundo o sexo. Marília, SP, Brasil, 2016

\begin{tabular}{|c|c|c|c|}
\hline \multirow{2}{*}{ Variáveis } & Masculino - 47 & Feminino - 91 & \multirow{2}{*}{$\mathbf{p}$} \\
\hline & $\mathrm{N}(\%)$ & $\mathrm{N}(\%)$ & \\
\hline Idade (anos) & & & 0,53 \\
\hline $60-69$ & $28(59,6)$ & $63(69,2)$ & \\
\hline $70-79$ & $14(29,8)$ & $21(23,1)$ & \\
\hline$\geq 80$ & $5(10,6)$ & $7(7,7)$ & \\
\hline Escolaridade & & & 0,39 \\
\hline Analfabeto & $7(14,9)$ & $17(18,7)$ & \\
\hline Fund. 1 incompleto & $22(46,8)$ & $30(33,0)$ & \\
\hline Fund. 1 completo e Fund. 2 incompleto & $12(25,5)$ & $25(27,5)$ & \\
\hline Outros & $6(12,8)$ & $19(20,9)$ & \\
\hline Estado conjugal & & & 0,06 \\
\hline Vive sem companheiro (a) & $19(40,4)$ & $52(57,1)$ & \\
\hline Vive com companheiro (a) & $28(59,6)$ & $39(42,9)$ & \\
\hline Religião & & & 0,02 \\
\hline Católica & $36(76,6)$ & $51(56,9)$ & \\
\hline Evangélica & $7(14,9)$ & $33(36,3)$ & \\
\hline Outras & $4(8,6)$ & $7(7,7)$ & \\
\hline Classificação econômica & & & 0,41 \\
\hline A & $1(2,1)$ & $2(2,2)$ & \\
\hline B & $3(6,4)$ & $9(9,9)$ & \\
\hline C & $26(55,4)$ & $59(64,9)$ & \\
\hline D & $17(36,2)$ & $20(22,0)$ & \\
\hline E & 00 & $1(1,1)$ & \\
\hline
\end{tabular}


Tabela 1 - Continuação

\begin{tabular}{lccc}
\hline \multirow{2}{*}{ Variáveis } & Masculino - 47 & Feminino - 91 & p \\
\cline { 2 - 3 } & $\mathbf{N}(\%)$ & $\mathbf{N}(\%)$ & 0,46 \\
\hline Idade da aposentadoria (anos) & & & \\
$\leq 50$ & $4(11,4)$ & $12(21,4)$ & \\
$51-60$ & $13(37,1)$ & $18(32,1)$ & 0,60 \\
$\geq 61$ & $18(51,4)$ & $26(46,4)$ & \\
Participação econômica na família & & & \\
$\quad$ Único responsável & $14(29,8)$ & $26(28,6)$ & 0,29 \\
Divide responsabilidade & $26(55,3)$ & $45(49,5)$ & \\
Outro & $7(14,9)$ & $20(22,0)$ & \\
Origem da renda & & $38(42,2)$ & $13(14,4)$ \\
$\quad$ Aposentadoria & $27(58,7)$ & $24(26,7)$ \\
Pensão & $4(8,7)$ & $15(16,7)$ \\
Outras & $8(17,4)$ & \\
Duas rendas ou mais & $7(15,2)$ & \\
\hline
\end{tabular}

Em relação à classificação econômica, observou-se predomínio da classe $\mathrm{C}$ em ambos os sexos. A maioria dos entrevistados encontrava-se aposentada. Predominantemente, após os 60 anos, a aposentadoria representava a principal fonte de renda e os entrevistados referiram que participavam economicamente, dividindo responsabilidade com outro membro da família.

Conforme se observa na Tabela 2, a maioria dos entrevistados apresentava fala espontânea e compreensão das perguntas. A maior parte classificou a saúde como boa ou regular. Em relação ao uso de álcool (consumo pregresso, frequência e quantidade), houve diferença estatisticamente significativa em relação ao sexo, com homens declarando o uso em maior porcentagem $(p=0,01)$. Constatou-se associação positiva entre sexo e vacinação contra a gripe $(p=0,05)$, sendo que as mulheres realizavam em maior proporção tais práticas. Não houve associação positiva na relação ao número de quedas e sexo.

Tabela 2 - Distribuição dos idosos usuários do Ambulatório de Saúde Mental, por sexo, de acordo com dados de condições de saúde. Marília, SP, Brasil, 2016

\begin{tabular}{|c|c|c|c|}
\hline \multirow{2}{*}{ Variáveis } & Masculino -47 & Feminino-91 & \multirow{2}{*}{$\mathbf{p}$} \\
\hline & $\mathbf{N}(\%)$ & $\mathbf{N}(\%)$ & \\
\hline Fala espontânea* & $36(76,6)$ & $81(89,0)$ & 0,23 \\
\hline Compreensão* & $36(76,6)$ & $80(87,9)$ & 0,21 \\
\hline Como classifica a própria saúde & & & 0,67 \\
\hline Ótima & $1(2,1)$ & $1(1,1)$ & \\
\hline Boa & $14(29,8)$ & $27(29,7)$ & \\
\hline Regular & $22(46,8)$ & $40(44,0)$ & \\
\hline Ruim & $7(14,9)$ & $16(17,6)$ & \\
\hline Péssima & $2(4,3)$ & $7(7,7)$ & \\
\hline Não sabe & $1(2,1)$ & $0(0)$ & \\
\hline Álcool atualmente* & $6(12,8)$ & $15(16,5)$ & 0,56 \\
\hline Álcool anteriormente* & $36(76,6)$ & $36(39,6)$ & 0,01 \\
\hline Vacinação contra gripe* & $28(59,6)$ & $69(75,8)$ & 0,05 \\
\hline Número de quedas no último ano & & & 0,29 \\
\hline 1 & $5(10,6)$ & $20(22,0)$ & \\
\hline 2 & $5(10,6)$ & $7(7,7)$ & \\
\hline $3 \mathrm{ou}+$ & $7(14,9)$ & $17(18,7)$ & \\
\hline Nenhuma & $30(63,8)$ & $47(51,6)$ & \\
\hline Visão & & & 0,39 \\
\hline Cega/péssima/ruim & $14(29,8)$ & $21(23,1)$ & \\
\hline Regular & $10(21,3)$ & $29(31,9)$ & \\
\hline Boa & $23(48,9)$ & $41(45,1)$ & \\
\hline Audição & & & 0,94 \\
\hline Surdo/Ouve com muita dificuldade/Ouve com dificuldade & $6(12,7)$ & $10(11,0)$ & \\
\hline Ouve com alguma dificuldade & $7(14,9)$ & $13(14,3)$ & \\
\hline Sem problemas & $34(72,3)$ & $68(74,7)$ & \\
\hline
\end{tabular}


Tabela 2 - Continuação

\begin{tabular}{|c|c|c|c|}
\hline \multirow{2}{*}{ Variáveis } & Masculino-47 & Feminino - 91 & \multirow{2}{*}{$\mathbf{p}$} \\
\hline & $\mathrm{N}(\%)$ & $\mathrm{N}(\%)$ & \\
\hline Mastigação - problemas & & & 0,23 \\
\hline Nunca/Raramente & $38(61,7)$ & $83(65,9)$ & \\
\hline Frequentemente/Sempre & $8(17,0)$ & $7(7,7)$ & \\
\hline Não sabe & $1(2,1)$ & $1(1,1)$ & \\
\hline Uso de aparelhos & & & 0,02 \\
\hline Óculos & $10(21,3)$ & $8(8,8)$ & \\
\hline Uso de prótese & $6(12,8)$ & $12(13,2)$ & \\
\hline Outros & $2(4,3)$ & $4(4,4)$ & \\
\hline 2 ou mais & $21(44,7)$ & $63(69,2)$ & \\
\hline Não usa & $8(17,0)$ & $4(4,4)$ & \\
\hline Grau de dependência & & & 0,02 \\
\hline Dependência & $12(25,5)$ & $13(14,3)$ & \\
\hline Dependência parcial & $17(36,2)$ & $20(22,0)$ & \\
\hline Independência & $18(38,3)$ & $58(63,7)$ & \\
\hline
\end{tabular}

*Variáveis dicotômicas (com respostas sim ou não). Descreveu-se apenas o número que representa a resposta sim

Quanto aos órgãos dos sentidos, homens e mulheres, em maioria, avaliaram a visão como boa ou regular, negaram problemas de audição e nunca ou raramente tiveram problemas com a mastigação. Apareceu associação positiva entre o uso de aparelhos e o sexo $(p=0,02)$. Os aparelhos mais frequentemente utilizados eram óculos e prótese dentária. Para atividades instrumentais da vida diária, encontrou-se associação positiva entre o parâmetro e sexo $(p=0,02)$, sendo que mulheres apresentaram maior independência em relação aos homens.

Acerca dos problemas de saúde referidos, que constam na Tabela 3, as mulheres foram as que mais referiram a presença de reumatismo $(p=0,03)$, obesidade $(p=0,01)$ e hipotensão ortostática $(p=0,03)$. A insônia foi o problema de saúde mais referido, seguido pela hipertensão arterial e o diabetes, em ambos os sexos.

No tocante ao diagnóstico de transtorno mental, entre os homens, houve predomínio de transtornos mentais orgânicos, incluindo sintomáticos; transtornos mentais e de comportamento decorrentes do uso de substâncias psicoativas e transtornos neuróticos, como os transtornos de ansiedade generalizada.

A porcentagem de idosos com diagnósticos pertencentes aos grupos das esquizofrenias (F20-F29) foi semelhante em ambos os sexos. Entre as mulheres, foram mais frequentes os diagnósticos de transtornos de humor e de personalidade. As mulheres tratadas nesse ambulatório de saúde mental tiveram a maioria dos diagnósticos na idade adulta $(59,3 \%)$ e os homens foram diagnosticados com sessenta anos ou mais $(61,7)$, diferindo estatisticamente $(p=0,01)$.

Em relação às internações, $25,4 \%$ tiveram uma internação e 13,8\%, duas internações no último ano. Sobre os idosos entrevistados, constatou-se que mais da metade frequentava somente o ambulatório de saúde mental. A grande maioria, em ambos os sexos, nunca fez tratamento psicoterápico (Tabela 4).

Tabela 3 - Problemas de saúde referidos pelos idosos atendidos do Ambulatório de Saúde Mental segundo o sexo. Marília, SP, Brasil, 2016

\begin{tabular}{|c|c|c|c|}
\hline \multirow{2}{*}{ Variáveis* } & Masculino - 47 & Feminino - 91 & \multirow{2}{*}{ p } \\
\hline & $\mathrm{N}(\%)$ & $\mathrm{N}(\%)$ & \\
\hline Reumatismo & $1(2,1)$ & $12(13,2)$ & 0,03 \\
\hline Asma & $6(12,8)$ & $10(11,0)$ & 0,76 \\
\hline Má circulação & $8(17,0)$ & $26(28,6)$ & 0,14 \\
\hline Diabetes Mellitus & $18(38,3)$ & $34(37,4)$ & 0,91 \\
\hline Hipertensão & $28(59,6)$ & $54(59,3)$ & 0,90 \\
\hline Obesidade & $2(4,3)$ & $22(24,2)$ & 0,01 \\
\hline Derrame & $4(12,8)$ & $9(11,0)$ & 0,53 \\
\hline Incontinência urinária & $14(29,8)$ & $19(20,9)$ & 0,24 \\
\hline Insônia & $26(55,3)$ & $57(62,6)$ & 0,40 \\
\hline Catarata & $2(4,3)$ & $10(11,0)$ & 0,16 \\
\hline Coluna & $6(12,8)$ & $21(23,1)$ & 0,15 \\
\hline Hipotensão ortostática & $0(0)$ & $8(8,8)$ & 0,03 \\
\hline
\end{tabular}

*Variáveis dicotômicas (com respostas sim ou não). Descreveu-se apenas o número que representa a resposta sim 
Tabela 4 - Diagnóstico do transtorno mental dos idosos atendidos do Ambulatório de Saúde Mental segundo o sexo. Marília, SP, Brasil, 2016

\begin{tabular}{|c|c|c|c|}
\hline Variáveis & Masculino $\mathrm{N}=47$ (\%) & Feminino $\mathrm{N}=91(\%)$ & $\mathrm{p}$ \\
\hline Diagnóstico psiquiátrico (CID 10) & & & 0,03 \\
\hline Transtornos mentais orgânicos, incluindo os sintomáticos & $13(27,7)$ & $10(11,0)$ & \\
\hline Transtornos mentais decorrentes de substâncias psicoativas & $3(6,4)$ & $2(2,2)$ & \\
\hline Esquizofrenia, transtornos esquizotípicos e delirantes & $10(21,3)$ & $19(20,9)$ & \\
\hline Transtornos de humor & $12(25,5)$ & $37(40,7)$ & \\
\hline Transtornos neuróticos & $6(12,8)$ & $8(8,8)$ & \\
\hline Síndromes comportamentais associadas a perturbações fisiológicas & $0(0)$ & $1(1,1)$ & \\
\hline Transtornos de personalidade e de comportamentos & $3(6,4)$ & $14(15,4)$ & \\
\hline Idade do primeiro diagnóstico & & & 0,01 \\
\hline$\leq 9$ anos - Infância & $0(0)$ & $1(1,1)$ & \\
\hline 9 - 24 - Adolescência & $4(8,5)$ & $5(5,5)$ & \\
\hline $25-59$ - Adulto & $13(27,7)$ & $54(59,3)$ & \\
\hline 60 anos ou mais - Idoso & $29(61,7)$ & $31(34,1)$ & \\
\hline Não soube informar & $1(2,1)$ & $0(0)$ & \\
\hline Número de internações no último ano & & & 0,54 \\
\hline 1 & $15(31,9)$ & $20(22,0)$ & \\
\hline 2 & $5(10,6)$ & $14(15,4)$ & \\
\hline 3 ou mais & $4(8,5)$ & $6(6,6)$ & \\
\hline Não internou & $23(48,9)$ & $51(56,0)$ & \\
\hline Fez tratamento psicoterápico & & & 0,16 \\
\hline Sim & $2(4,3)$ & $10(11,0)$ & \\
\hline Não & $45(95,7)$ & $81(89,0)$ & \\
\hline
\end{tabular}

\section{Discussão}

Neste estudo, foram entrevistados 138 idosos, sendo que a maioria, tanto de homens como de mulheres, encontrava-se na faixa dos 60 a 69 anos de idade. Outro estudo encontrou dados semelhantes, além de confirmar que, nessa faixa de idade, os idosos apresentam autopercepção de saúde melhor em relação a idosos de outras faixas etárias ${ }^{(10)}$.

Dos idosos entrevistados, a maioria era mulher, achado semelhante ao encontrado em outro estudo também realizado em ambulatório de saúde mental(11). Nesse sentido, dados do IBGE, de 2018, expressam o crescente envelhecimento da população brasileira, sendo que, dos indivíduos acima de 60 anos, 8,6\% são mulheres e $6,8 \%$, homens(12), o que aponta para a feminilização da velhice ${ }^{(13)}$.

As mulheres são as principais consumidoras dos serviços ambulatoriais, uma vez que apresentam maior facilidade para expressar aflições e procurar por serviços médicos para queixas do ponto de vista emocional ou físico(14).

Mulheres apresentaram maior porcentagem para o estado conjugal "vive só", não sendo raro o abandono por parte do marido, no caso da doença mental, questão também apontada como desvantagem ao cuidado da mulher, pois, quando o homem adoece psiquicamente, a esposa tende a continuar ao lado deste ${ }^{(15)}$.

Além disso, para explicar o fato de que, entre as mulheres, prevalece a condição de viver sem o companheiro, outras variáveis podem ser consideradas, como a expectativa de vida, que, em 2012, era de 71 anos para o sexo masculino e 74,6 para o sexo feminino. Já que homens morrem mais cedo, existe número considerável de mulheres viúvas ${ }^{(16)}$ que, frequentemente, podem optar pela vida sem novo companheiro por ainda se submeterem às normas e regras sociais ou porque a família não declara apoio para novos relacionamentos amorosos.

Em relação à saúde mental e ao sexo, ao considerar a característica feminina de prestadora de cuidados informais à saúde, cabe o questionamento sobre as condições da mulher que precisa de assistência psiquiátrica e demanda cuidados. Solteiras ou sem filhos, em situação de sofrimento mental, apresentam características contrárias àquelas socialmente esperadas, mais vinculadas ao exercício do papel de esposa e mãe ${ }^{(14)}$. Observa-se que a sociedade mantém a cobrança da função materna, entretanto, algumas condições prejudicam esta execução(17).

A religião católica foi referida pela maioria, indo ao encontro do censo demográfico de $2010^{(16)}$.

Ao ponderar a totalidade da população brasileira de idosos, cerca de metade tem menos de quatro anos de estudo. A escolaridade baixa, principalmente entre mulheres, pode ser atribuída às características da sociedade e às políticas de educação prevalecentes na década de 1940, quando o acesso à educação era, ainda, muito restrito. Em 2000, o número médio de anos de escolaridade dos idosos responsáveis pelos domicílios 
era de 3,5 anos para homens e ainda menos para as mulheres, que apresentavam 3,1 anos. Neste sentido, os dados obtidos neste estudo não diferem dos da população brasileira $^{(18)}$. Importante considerar que baixa escolaridade e renda significa menor acesso aos serviços básicos ${ }^{(19)}$.

Os idosos pesquisados pertenciam predominantemente à classe econômica $\mathrm{C}$, dado semelhante a estudo de base populacional realizado em município do interior do Rio Grande do Sul, Brasil, com 1.448 idosos, o qual mostrou que $56,8 \%$ dos entrevistados pertenciam a esta classe econômica(20).

A maioria dos idosos entrevistados encontrava-se aposentada após os sessenta anos. Este dado vai ao encontro dos indicadores sociais para aposentados ou pensionistas na Região Sudeste(18). Estudo realizado na Universidade Federal do Rio Grande do Norte, entre 2002-2005, avaliou servidores aposentados por transtornos mentais e constatou que 35\% se aposentaram com idades entre 50 e 59 anos. A antecipação da aposentadoria pode ocorrer por essas pessoas apresentarem sofrimento psíquico anterior à velhice, carregando anos de incapacitação, o que afeta o trabalho(21).

No que se refere à origem da renda, dados do IBGE corroboram os obtidos neste estudo: homens idosos têm renda que se origina principalmente de aposentadoria $(58,7 \%)$ e mulheres têm renda oriunda tanto de aposentadorias $(42,2 \%)$, pensão $(14,4 \%)$ ou de outra fonte de renda $(26,7 \%)^{(22)}$.

Aproximadamente, a metade dos idosos estudados referiu participar economicamente, dividindo responsabilidades com outros membros da família. Apesar de os idosos em questão serem usuários do Sistema Único de Saúde (SUS), informaram que grande parte dos gastos era com a saúde (31,9\% dos homens e $37,4 \%$ das mulheres). Esta informação, quando associada a outras características sociodemográficas dos idosos, como a fonte de renda e a classe social, pode inferir que os idosos com transtornos mentais que utilizam o ambulatório contam com poucos recursos financeiros para manter uma condição de vida satisfatória às necessidades.

Entre os idosos entrevistados, poucos foram aqueles que não conseguiram se comunicar verbalmente com clareza, mesmo considerando que alguns transtornos mentais trazem consigo dificuldades na compreensão e verbalização de palavras de forma espontânea, demandando ajuda de outras pessoas para apoiar a comunicação.

O uso pregresso de álcool mostrou associação positiva com o sexo $(p=0,001)$, sendo os homens declararam mais essa prática. Estudo descritivo, com dados da Pesquisa Nacional de Saúde (PNS), referentes ao consumo abusivo de álcool nos 30 dias anteriores à entrevista, mostrou que a prevalência foi de $13,7 \%$, sendo superior entre homens $(21,6 \%)$ em relação às mulheres $(6,6 \%)$, com predomínio em jovens ${ }^{(23)}$. Segundo estudo realizado em dois CAPS AD na cidade de Florianópolis/SC, Brasil, na idade adulta, o diagnóstico de dependência de álcool difere na proporção de 8,5:1 e 5,5:1 entre homens e mulheres, respectivamente ${ }^{(24)}$.

Pesquisa realizada em Portugal, com idosos atendidos em Unidade de Saúde da Família, evidenciou presença significativa do uso de álcool, com prevalência, também, em homens (56,2\%). Além disso, um terço da amostra apresentava consumo de risco, definido por aquele superior ou igual a 14 unidades-padrão de etanol (168g) por semana(25).

Em relação à vacinação contra a gripe, também houve associação estatisticamente significativa em relação ao sexo, sendo que as mulheres aderiram mais do que os homens a tal prática. Isto confirma o padrão encontrado na população feminina sobre maior busca por cuidados e prevenção de saúde. No Rio Grande do Sul, Brasil, também se comprovou que as mulheres idosas adotam comportamentos saudáveis e medidas de prevenção de riscos à saúde mais frequentemente do que os homens, uma vez recebem orientações sobre hábitos saudáveis com maior frequência(26).

O grau de dependência para as atividades de vida diárias que representa importante indicativo das condições de vida e saúde dos idosos foi maior entre os homens. No entanto, a diversidade de instrumentos utilizados para essa avaliação e o fato de ela ser realizada, principalmente, no contexto institucional, dificultam qualquer tentativa de comparação dos dados.

No concernente aos problemas de saúde, os idosos com transtornos mentais seguem o padrão de alta prevalência de doenças crônicas não transmissíveis, especialmente hipertensão arterial e diabetes, o que contribui para o agravo ao estado de saúde, visto que são doenças que apresentam elevado risco de complicações cardiocirculatórias e acarretam o uso de grande número de medicamentos, o que pode interferir na autonomia e qualidade de vida, principalmente, ao se considerar que tais problemas se somam ao transtorno mental(27).

Além disso, a maioria dos entrevistados apontou a presença de insônia, apesar de fazer acompanhamento no ambulatório de saúde mental e uso de psicofármacos. Dados da literatura apontam que $44,9 \%$ dos idosos possuem algum distúrbio do sono, sendo a maior frequência em mulheres $(51,5 \%)$ e trazem, enquanto fatores associados, sexo, dor, incontinência urinária e noctúria(28).

Obesidade tem sido o problema mais frequente entre as mulheres, quando comparadas com os homens. Revisão da literatura sobre o tema evidenciou que as mulheres apresentam índices de obesidade próximos ao dobro em relação aos dos homens, sendo a faixa etária mais crítica dos 60 aos $69 \operatorname{anos}^{(29)}$. Constata-se, 
também, a existência de associações positivas entre a obesidade e as perturbações do humor e a ansiedade ${ }^{(30)}$.

Coincide com os resultados deste estudo o fato de que, nos homens, prevalecem os transtornos mentais e de comportamento decorrentes do uso de substâncias psicoativas e, nas mulheres, aqueles relacionados aos transtornos de humor e personalidade(31). Distintas teorias explicam as diferenças de prevalência de doenças mentais entre homens e mulheres, sendo que as mesmas relacionam tais diferenças tanto a questões internas como externas, destacando-se, especialmente, as funções sociais relacionados ao sexo, com a indicação de que se trata de rede multifatorial de difícil compreensão(32).

Este artigo evidenciou as diferenças entre os sexos, destacando questões que se referem ao desafio de compreender o mundo onde o indivíduo se insere, conhecendo o passado que o influenciou e motivou o adoecimento e as condições do envelhecimento(33).

Por fim, considera-se como limite do estudo o fato de os dados terem sido coletados apenas no ambulatório de saúde mental de uma instituição de ensino, o que pode não representar a totalidade de idosos com transtornos mentais, visto que, em outros cenários importantes, como o CAPS e os serviços de atenção hospitalar, estes possam apresentar condições distintas, por vezes, com problemas mais intensos.

Compreende-se, portanto, a necessidade de estudos mais aprofundados que, além de revelar as diferenças entre os sexos, possam demonstrar, de forma mais direta, as necessidades específicas de idosos com transtornos mentais.

\section{Conclusão}

Neste estudo, foi possível evidenciar diferenças significativas entre condições de vida e saúde e sexo de idosos atendidos em ambulatório de saúde mental. As mulheres demonstraram maior aceitação da vacina contra gripe e utilizaram, em maior proporção, aparelhos, como óculos e próteses dentárias, quando necessários; tenderam ao estado conjugal "vive só" e maior independência nas atividades de vida diária. Os homens tiveram diagnósticos em saúde mental mais tardios após os sessenta anos. Quase a totalidade fez uso pregresso de álcool e apresentou maior dependência para atividades instrumentais de vida diária.

Os achados sinalizam a importância de ampliar o conhecimento acerca das necessidades de idosos com transtornos mentais, com medidas assistenciais e políticas voltadas ao tratamento precoce, a fim de minimizar o impacto da doença mental no processo de envelhecimento. É preciso reconhecer e atender às especificidades inerentes ao sexo, o que implica perspectiva ampliada e contextualizada que não se restringe apenas a essa variável. O acesso ao tratamento especializado em saúde mental, nos diversos ciclos da vida, torna-se, nesse cenário, essencial para manter condições adequadas de saúde no envelhecimento.

\section{Referências}

1. Andreas S, Schulz H, Volkert J, Dehoust M, Sehner S, Suling $A$, et al. Prevalence of mental disorders in elderly people: the European MentDis_ICF65+ study. Br J Psychiatry. 2017 Feb;210(2):125-31. doi: http://dx.doi.org/10.1192/bjp.bp.115.180463.

2. Onofri Junior VA, Martins VS, Marin MJS. Elderly health care in the Family Health Strategy and the prevalence of common mental disorders. Rev Bras Geriatr Gerontol. 2016;19(1):21-33. doi: http://dx.doi.org/ 10.1590/1809-9823.2016.15004

3. Ministério da Saúde (Brasil). Portaria n. 3088 de 23 de dezembro de 2011. Institui a Rede de Atenção Psicossocial para pessoas com sofrimento ou transtorno mental e com necessidades decorrentes do uso de crack, álcool e outras drogas, no âmbito do Sistema Único de Saúde. Diário Oficial da União, Brasília (DF); 30 dez 2011; Seção 1: 59-61.

4. Weber CAT. Rumos da saúde mental no brasil após 1980. Rev Debates Psiquiatr. 2013 [citado 13 jan 2019]; 3(3):14-22. Disponível em: http://www.apalweb.org/ docs/revista15abp_.pdf

5. Graham N, Lindesay J, Katona C, Bertolote JM, Camus V, Copeland JRM, et al. Redução da estigmatização e da discriminação das pessoas idosas com transtornos mentais: uma declaração técnica de consenso. Rev Psiquiatr Clín. 2007;34(1):39-49. doi: http://dx.doi.org/ 10.1590/S0101-60832007000100006

6. Zanello V, Fiuza G, Costa HS. Saúde mental e gênero: facetas gendradas do sofrimento psíquico. Fractal (Niterói). 2015 [citado 19 dez 2018];27(3): 238-46. Disponível em: http://www.scielo.br/pdf/fractal/ v27n3/1984-0292-fractal-27-3-0238.pdf

7. Cerqueira MB, Pupo LR. Condições e modos de vida em duas favelas da baixada santista e suas interfaces com o acesso aos serviços de saúde. Rev Baiana de Saúde Pública. 2009 [citado 19 dez 2018]; 33(2):68-84. Disponível em: http://files.bvs.br/ upload/S/0100-0233/2009/v33n2/a007.pdf

8. Lebrão $M L$, Laurenti R. Saúde, bem-estar e envelhecimento: o estudo SABE no Município de São Paulo. Rev Bras Epidemiol. 2005;8(2):127-41. doi: http://dx.doi.org/10.1590/S1415-790X2005000200005 9. Apóstolo JLA. Instrumentos para avaliação em geriatria (geriatric instruments) [dissertação da Internet]. Coimbra: Escola Superior de Enfermagem de Coimbra; 2011. 118p. [citado 15 out 2016]. Disponível em: https://web.esenfc.pt/v02/include/download. php?id_ficheiro $=20538 \&$ codigo $=688697509$ 
10. Santos EC, Couto BM, Bastone AC. Fatores associados à autoavaliação negativa da saúde em idosos cadastrados nas Unidades Básicas de Saúde. ABCS Health Sci. 2018; 43(1). doi: https://dx.doi.org/ 10.7322/abcshs.v43i1.999

11. Naloto DCC, Lopes FC, Barberato FS, Lopes LC, Del Fiol FS, Bergamaschi CC. Prescription of benzodiazepines for adults and older adults from a mental health clinic. Ciênc Saúde Coletiva. 2016;21(4):1267-76. doi: http://dx.doi.org/ 10.1590/1413-81232015214.10292015

12. Instituto Brasileiro de Geografia e Estatística. IBGEEduca. Conheça o Brasil - População Quantidade de homens e mulheres. Rio de Janeiro: IBGE [online]; 2018 [citado 23 jun 2019]. Disponível em: https://educa. ibge.gov.br/jovens/conheca-o-brasil/populacao/18320quantidade-de-homens-e-mulheres.html

13. Lins IL, Andrade LVR. A feminização da velhice: representação e silenciamento de demandas nos processos conferencistas de mulheres e pessoas idosas. Mediações. 2018;23(3):436-65. doi: http://dx.doi.org/ 10.5433/2176-6665.2018v23n3p436

14. Pegoraro RF, Caldana RHL. Mulheres, loucura e cuidado: a condição da mulher na provisão e demanda por cuidados em saúde mental. Saúde Soc. 2008;17(2):82-94. doi: http://dx.doi.org/10.1590/S0104-12902008000200009

15. Souza M, Marcon SS, Bueno SMV, Carreira L, Baldissera VDA. A vivência da sexualidade por idosas viúvas e suas percepções quanto à opinião dos familiares a respeito. Saúde Soc. 2015;24(3):936-44. doi: http:// dx.doi.org/10.1590/S0104-12902015132060

16. Annes LMB, Mendonça HGS, Lima FM, Lima MDAS, Aquino JM. Perfil sociodemográfico e de saúde de idosas que participam de grupos de terceira idade em Recife, Pernambuco. Revista Cuidarte [Internet]. 2017;8(1):1499-508. Recuperado de: http://www. redalyc.org/articulo.oa?id=359549159009

17. Trindade V, Bartilotti C. "Não quebrou a corrente, mas abriu um elo entre nós": o impacto da dependência química materna sobre o vínculo mãe-filho. SMAD, Rev Eletrônica Saúde Mental Álcool Drog. 2017;13(1):4-12. doi: https://doi.org/10.11606/issn.1806-6976.v13i1p4-12 18. Instituto Brasileiro de Geografia e Estatística. Pesquisa Nacional por Amostra de Domicílios Contínua 2012-2013 (PNAD). Rio de Janeiro: IBGE [online]; 2013 [citado 13 dez 2018]. Disponível em: https://biblioteca.ibge.gov.br/visualizacao/ periodicos/2421/pnact_2013_2tri.pdf

19. Melo NCV, Ferreira MAM, Teixeira KMD. Condições de vida dos idosos no Brasil: uma análise a partir da renda e nível de escolaridade. Oikos: Rev Bras Economia Doméstica. 2014 [acesso em 16 dez 2018];25(1):4-19. Disponível em: http://www.locus.ufv.br/bitstream/ handle/123456789/13829/154-953-1-PB.pdf?sequence $=1$
20. Vieira LS, Gomes AP, Bierhals IO, Farías-Antúnez S, Ribeiro CG, Miranda $V$ et al. Quedas em idosos no Sul do Brasil: prevalência e determinantes. Rev Saúde Pública. 2018:52. doi: http://DOI: 10.11606/ S1518-8787.2018052000103

21. Miranda FAN, Carvalho GRP, Fernandes RL, Silva MB, Sabino MGG. Saúde mental, trabalho e aposentadoria: focalizando a alienação mental. Rev Bras Enferm. 2009;62(5):711-6. doi: http://dx.doi.org/ 10.1590/S0034-71672009000500010

22. Instituto Brasileiro de Geografia e Estatística. Censo demográfico 2010: características gerais da população, religião e pessoas com deficiência. Rio de Janeiro: IBGE [online]; 2012 [citado 13 dez 2018]. Disponível em: http://biblioteca.ibge.gov.br/visualizacao/periodicos/94/ cd_2010_religiao_deficiencia.pdf

23. Garcia LP, Freitas LRS. Consumo abusivo de álcool no Brasil: resultados da Pesquisa Nacional de Saúde 2013. Epidemiol Serv Saúde. 2015;24(2). doi: https:// doi.org/10.5123/S1679-49742015000200005

24. Lopes MA, Sprícigo JS, Mitjavila MR, Schneider DR, Abreu, D. As diferenças de idade e gênero entre usuários de CAPS ad e as implicações na rede de atenção. SMAD, Rev. Eletrônica Saúde Mental Álcool Drog. 2018; 14(3):159-67. doi: http://dx.doi.org/10.11606/ issn.1806-6976.smad.2018.000412.

25. Martins A, Parente J, Araújo J, Menezes MJ. Prevalência do consumo de risco de álcool no idoso: estudo numa unidade dos cuidados primários da região de Braga. Rev Port Med Geral Fam. 2016;32(4):270-4. 26. Flores TR, Nunes BP, Assunção MCF, Bertoldi AD. Hábitos saudáveis: que tipo de orientação a população idosa está recebendo dos profissionais de saúde? Rev Bras Epidemiol. 2016; 19(1). doi: https://doi. org/10.1590/1980-5497201600010015

27. Brito AMM, Camargo BV. Representações sociais, crenças e comportamentos de saúde: um estudo comparativo entre homens e mulheres. Temas Psicol. 2011 [citado 08 dez 2018];19(1):283-303. Disponível em: http://pepsic.bvsalud.org/pdf/tp/v19n1/v19n1a23.pdf 28. Moreno CRC, Santos JLF, Lebrão ML, Ulhôa MA, Duarte YAO. Problemas de sono em idosos estão associados a sexo feminino, dor e incontinência urinária. Rev. Bras. Epidemiol. 2019 [Citado 23 jun 2019];21(02). doi: https://doi.org/10.1590/1980-549720180018.supl.2 29. Scheidt A, Liberali R, Navarro F. Prevalência de obesidade na terceira idade. Revista Brasileira de Obesidade, Nutrição e Emagrecimento. 2009 [citado 04 jan 2019];3(15):234-40. Disponível em: http://www. rbone.com.br/index.php/rbone/article/viewFile/152/149 30. Pereira C, Brandão I. Uma perspectiva da psicopatologia da obesidade. Arquivos de Medicina. 2014 [acesso em 07 dez 2018];28(5):152-159. Disponível em: http://www.scielo.mec.pt/pdf/am/v28n5/v28n5a04.pdf 
31. Campos, I.; Zonello, V. Saúde mental e gênero: o sofrimento psíquico e a invisibilidade das violências mental. Vivência: Revista de Antropologia. 2016 [citado 11 jan 2019]; 1(48):105-17. Disponível em: https://periodicos. ufrn.br/vivencia/article/view/11505/8096

32. Rabasquinho C, Pereira H. Gênero e saúde mental: uma abordagem epidemiológica. Analise Psicologica. 2007 [citado 15 dez 2018];3(25):439-54. Disponível em: http://www.scielo.mec.pt/pdf/aps/v25n3/v25n3a10.pdf 33. Villar L, Margarita A, A saúde mental dos indivíduos na atualidade. Rev. Eletrônica Saúde Mental Álcool Drog. 2017;13(1):1-3. doi: https://doi.org/10.11606/ issn.1806-6976.v13i1p1-3

\section{Contribuição dos autores}

Concepção e planejamento do estudo: Maria Cássia Corrêa Mazzi Freire, Maria José Sanches Marin, Carlos Alberto Lazarini. Obtenção, análise e interpretação dos dados: Maria Cássia Corrêa Mazzi Freire, Maria José Sanches Marin, Carlos Alberto Lazarini. Análise estatística: Maria Cássia Corrêa Mazzi Freire, Maria José Sanches Marin, Carlos Alberto Lazarini. Preparação e redação do manuscrito: Maria Cássia Corrêa Mazzi Freire, Maria José Sanches Marin, Carlos Alberto Lazarini, Daniela Garcia Damasceno. Revisão crítica do manuscrito: Maria José Sanches Marin, Carlos Alberto Lazarini, Daniela Garcia Damasceno.

Todos os autores aprovaram a versão final do texto.

Conflito de interesse: Os autores declaram não haver conflito de interesse. 